\title{
Ontologia de aplicação no domínio de mortalidade: uma ferramenta de apoio para o preenchimento da declaração de óbitos
}

\author{
Fabricio Martins Mendonça \\ Mestre em informática pela Pontifícia Universidade Católica \\ de Minas Gerais, Belo Horizonte, MG - Brasil. Professor da \\ Faculdade Pitágoras Núcleo Computação e Engenharia - \\ Belo Horizonte, MG - Brasil \\ E-mail: fabriciommendonca@gmail.com
}

\author{
Ana Maria Pereira Cardoso \\ Pós-doutora em ciência da informação pela Universidade \\ de Évora - Portugal. Professora da Pontifícia Universidade \\ Católica de Minas Gerais, Instituto de Informática - Belo \\ Horizonte, MG - Brasil \\ E-mail: anacard@pucminas.br
}

\section{Eliane Drumond}

Doutora em saúde pública, epidemiologia pela Universidade Federal de Minas Gerais, UFMG, Brasil. Técnica em saúde da Secretaria Municipal de Saúde e Assistência de Belo Horizonte - Belo Horizonte, MG - Brasil

E-mail: elianefd@pbh.gov.br

\section{Resumo}

Os dados do Sistema de Informação sobre Mortalidade (SIM) representam a principal fonte de informações sobre mortalidade no Brasil, embora apresentem com frequência alguma inconsistência. Um dos principais problemas na geração de dados confiáveis sobre mortalidade no Brasil decorre das dificuldades enfrentadas pelos médicos para o correto preenchimento da Declaração de Óbitos (DO), documento padrão de alimentação de dados para o SIM. O artigo apresenta uma ferramenta de apoio para o correto preenchimento da $\mathrm{DO}$, representada por meio de uma ontologia de aplicação desenvolvida com a participação de profissionais de saúde especialistas no domínio de mortalidade. A ferramenta foi disponibilizada aos médicos da Secretaria Municipal de Saúde e Assistência de Belo Horizonte (SMSA-BH) por meio de um site contendo os termos definidos na ontologia e seus relacionamentos, dispostos numa estrutura taxonômica. A solução proposta tem como objetivo principal auxiliar os médicos em sua tarefa de registrar os óbitos ocorridos, a partir do preenchimento correto da DO.
Palavras-chave

Declaração de Óbitos. Registros de Mortalidade. Ontologia. Sistemas de Informação.

\section{Ontology of application into the domain of mortality: a tool support for filling out the Death Certificate}

\begin{abstract}
Data from the Mortality Information System (SIM in Portuguese) are the main source of information on mortality in Brazil, in spite of frequent inconsistencies. One of the main drawbacks in the generation of reliable data on mortality in Brazil is due to the difficulties faced by doctors for the correct completion of the Death Certificate (DO in Portuguese). This standard document feeds data into the SIM. The paper presents a support tool for the correct completion of the $\mathrm{DO}$, represented by an application ontology, developed on the field of mortality as. The tool was made available to physicians of City Department of Health and Welfare of Belo Horizonte (SMSA-BH in Portuguese) via a website, containing the terms defined in the ontology and their relationships, arranged in a taxonomic structure. The proposed solution has the main objective of assisting doctors in their task of registering the occurred deaths, by means of the correct way of filling out the DO.
\end{abstract}

Keywords

Death Certificates. Mortality Registries. Ontology. Information Systems.

\section{INTRODUÇÃO}

A organização e o gerenciamento de informações são medidas imprescindíveis em qualquer domínio, mas ganham importância mais elevada quando se trata da área de saúde. 
Atualmente, uma estratégia que vem sendo utilizada e bem aceita em domínios da área de saúde para representação e organização do conhecimento especializado é o uso de ontologias. Ontologia pode ser entendida como artefato computacional para representação do conhecimento de domínios específicos ou genéricos.

Neste trabalho, pretende-se utilizar as possíveis contribuições de uma ontologia - colaboração e informação, compartilhamento, padronização, de um domínio da área de saúde constituído pelas informações sobre mortalidade no Brasil, diante de sua relevância para os estudos epidemiológicos e demográficos em nosso país.

Informações sobre mortalidade de um país são importantes não apenas para estudos epidemiológicos e demográficos da população, mas também para o planejamento e gestão de políticas e ações em saúde. Assim, torna-se imprescindível que as informações relacionadas a esse domínio sejam fidedignas, tempestivas e acessíveis.

Atualmente, os dados sobre mortalidade da população brasileira são produzidos tanto pelo Instituto Brasileiro de Geografia e Estatística (IBGE), quanto pelo Sistema de Informação de Mortalidade (SIM) do Ministério da Saúde, implantado no país a partir de 1975.

Embora o SIM represente a principal fonte de dados sobre mortalidade no Brasil, com visível aumento anual de sua abrangência, ainda assim enfrenta obstáculos na oferta de dados com maior qualidade, principalmente em razão do preenchimento inadequado do seu documento padrão, a Declaração de Óbito (DO) que é "documento-base do Sistema de Informação de Mortalidade (SIM) do Ministério da Saúde, utilizado pelos Cartórios para emissão da Certidão de Óbito", segundo definição do Manual de Procedimentos do SIM (BRASIL, 2001).

Sendo a DO o instrumento padronizado pelo Ministério da Saúde para a coleta de dados de confiabilidade - para representar o conhecimento

mortalidade no Brasil pelo SIM, é importante a realização de estudos e a proposição de soluções que possam contribuir para o aprimoramento de seu preenchimento, com os consequentes reflexos sobre a consistência das informações sobre mortalidade no país.

A partir de um estudo de caso ${ }^{1}$ sobre os problemas de preenchimento da DO realizado com o apoio de profissionais das áreas de saúde e epidemiologia da Secretaria Municipal de Saúde e Assistência de Belo Horizonte (SMSA/PBH-BH), Minas Gerais, especialistas no contexto das informações sobre mortalidade, tornou-se possível o desenvolvimento de uma ontologia de aplicação ${ }^{2}$ para representar o conhecimento do domínio de mortalidade.

O presente artigo apresenta a ontologia desenvolvida no domínio de mortalidade, elaborada para funcionar como ferramenta de apoio ao correto preenchimento da DO pelos médicos - pessoas ética e juridicamente responsáveis pelas informações nela declaradas.

A ontologia foi desenvolvida utilizando o Método 101 (Noy e McGuinness, 2000), com algumas alterações ao longo dos passos previstos no mesmo, e o editor de ontologias Protégé. O desenvolvimento foi realizado em duas etapas distintas e os resultados dessa construção foram apresentados aos profissionais anteriormente mencionados para validação da ontologia e avaliação das possíveis contribuições que ela pode trazer aos médicos no ato do preenchimento da DO.

Para atingir tal propósito, a base de conhecimento da ontologia sobre mortalidade foi exposta por meio de um site $^{3}$ contendo os termos definidos na

\footnotetext{
1 MENDONÇA, F.M; DRUMOND,E.F; CARDOSO, A.M.P. Problemas no preenchimento da declaração de óbito: estudo exploratório. Revista Brasileira de Estudos da População v. 27, p. 285295, 2010.

${ }^{2}$ Ontologia de aplicação é aquela que descreve conceitos dependendo de um domínio e tarefa específicos, os quais são especializações das ontologias relacionadas; e tem como objetivo a implementação, a parte prática (GUARINO, 1998).

${ }^{3}$ Disponível no endereço: http://www.ontologiamortalidade.gratix. com.br/
} 
Ontologia de aplicação no domínio de mortalidade: uma ferramenta de apoio para o preenchimento da declaração de óbitos

ontologia e seus relacionamentos, dispostos numa estrutura taxonômica.

\section{PROBLEMAS NO PREENCHIMENTO}

Como já mencionado, o preenchimento incorreto da DO representa o maior obstáculo para o funcionamento do processo de coleta de dados do SIM, por vezes comprometendo a qualidade dos dados gerados por esse sistema.

As dificuldades relatadas pelos médicos para o preenchimento da DO geram registros incorretos, que se relacionam principalmente com a falta de informações sobre o diagnóstico do paciente, a presença de campos no formulário da DO que não estão claramente definidos e a ausência do prontuário do paciente, conforme pode ser comprovado no estudo realizado em Mendonça (2009).

Somam-se a essas dificuldades problemas de aceitabilidade do SIM, decorrentes, talvez, do fato de o médico estar mal instruído quanto à importância do correto preenchimento de todos os campos da DO, ou, até, de sua negligência ou descaso com tal incumbência.

As orientações sobre o preenchimento da DO podem ser encontradas tanto no documento Manual de Instruções para o Preenchimento da Declaração de Óbitos ${ }^{4}$ do Ministério da Saúde quanto no documento conhecido como Cartilha do CFM, criado em 2006 numa parceria entre o Ministério da Saúde, o Conselho Federal de Medicina e o Centro Colaborador da Organização Mundial de Saúde (OMS), conforme citado em Brasil (2006).

Ambos os documentos contêm instruções sobre o preenchimento dos campos da DO e abordam também as responsabilidades e condições em que ela deve ser emitida, sendo assim usados neste trabalho como fonte de consulta para o conhecimento das

\footnotetext{
${ }^{4}$ O Manual de Instruções para o Preenchimento da Declaração de Óbitos encontra-se disponível em: portal.saude.gov.br/portal/arquivos/ pdf/declaracao_obitos $\% 20 . p d f$
}

regras sobre o preenchimento da DO e mapeamento na ontologia desenvolvida.

Apesar de sua reconhecida importância, "a declaração de óbito, instrumento de coleta para os dados de mortalidade no Brasil, ainda é vista pelo médico apenas como uma exigência legal para o sepultamento, sendo raras vezes encarada como fonte geradora de dados sobre a saúde de uma população", como afirmam Niobey et al. (1990).

De maneira geral, “os médicos não se sentem responsáveis pelo preenchimento dos campos referentes ao nome do falecido, sua profissão, idade, residência; ficando para funcionários administrativos - ainda menos comprometidos - a tarefa de completar esses campos", conforme citam Niobey et al. (1990) em seu trabalho. Como esses campos são de informações gerais sobre o falecido, não se referindo aos termos técnicos da medicina, muitos médicos não se preocupam em preenchê-los. Porém eles são fundamentais para o correto preenchimento da DO e, consequentemente, contribuem para as informações epidemiológicas.

Os médicos preocupam-se apenas em preencher as informações relativas às causas e condições do óbito, que fazem parte do Bloco VI, conforme modelo do formulário da DO (Anexo I). Ainda assim os dados registrados nesses campos apresentam problemas de preenchimento, como pode ser comprovado por meio de trabalhos publicados na área. Mais detalhes sobre esse tema podem ser encontrados em Mendonça (2009).

\section{CONSTRUÇÃO DE ONTOLOGIAS}

Existem várias metodologias para construção de ontologias, da mesma forma que existe também uma série de ferramentas que auxiliam neste processo, como é demonstrado em Almeida e Bax (2003). Mesmo que não exista uma metodologia padrão para a construção de ontologias, alguns princípios são essenciais a qualquer metodologia. Entre eles, podem ser citados os mencionados por Gruber (1993): clareza, objetividade, coerência e 
extensibilidade; e também os citados por GómezPérez (1999): completude, implementação mínima e compromisso ontológico.

De acordo com tais princípios, foram propostas diversas metodologias para construção de ontologias. $\mathrm{Na}$ tabela 1, obtida em revisão de literatura, observam-se como algumas metodologias foram utilizadas na área de saúde no Brasil, as ferramentas utilizadas no processo de construção e uma breve descrição da ontologia pesquisada.

Analisando os dados da tabela 1, é possível perceber a utilização recorrente do editor de ontologias Protégé como ferramenta auxiliar no processo de construção. O Protégé é um editor de ontologias open-source ${ }^{5}$ e também um framework, baseado em conhecimento, atualmente muito utilizado pelos desenvolvedores acadêmicos, usuários corporativos e, de modo geral, por usuários especialistas num domínio específico, que pretendem representar seus conhecimentos através de uma ontologia. Uma de suas funções mais interessantes é a exportação da ontologia construída para linguagens web de representação do conhecimento, tais como RDFS, OWL e XML Schema, todas consideradas linguagenspadrão para este tipo de aplicação pelo World Wide Web Consortium (W3C).

Sobre as metodologias apresentadas na tabela 1 , percebe-se que o método 101 foi utilizado em dois trabalhos recuperados por esta revisão e é usado também em outros domínios do conhecimento para construção de ontologias, como, por exemplo, a ontologia para publicação científica proposta por Eluan e Fachin (2007).

Sugerido por Noy e McGuinness (2000), o método 101 foi elaborado a partir de uma experiência no desenvolvimento de uma ontologia para vinhos e alimentos, utilizando o editor de ontologias Protégé. O método prevê a construção de uma hierarquia de classes sobre o domínio estudado, bem como a definição de propriedades e instâncias para

${ }^{5}$ Disponível gratuitamente para download no endereço: http:// protege.stanford.edu/ Acesso em: 26 out. 2010. estas classes. Considerando essa metodologia de construção, pode-se afirmar que existe uma relação bem próxima do editor de ontologias Protégé com o método 101, o que os torna uma solução compatível para o processo de desenvolvimento de ontologias.

\section{METODOLOGIA}

A ontologia de aplicação no domínio de mortalidade apresentada neste artigo foi desenvolvida utilizando o Método 101 e o editor de ontologias Protégé. Esse desenvolvimento foi realizado em duas etapas.

O método utilizado supõe a sequência de sete passos para a construção de ontologias. No primeiro passo determinam-se o escopo e o domínio da ontologia, por meio de algumas questões sugeridas por Noy e McGuinness (2000):

- Qual o domínio que a ontologia deve cobrir?

- Para que alguém usará a ontologia?

- A que tipos de questões as informações contidas na ontologia devem responder?

- Quem usará e manterá a ontologia?

No segundo passo, os autores do método 101 ressaltam o valor de procurar trabalhos relacionados, e avaliam se eles podem ser refinados e estendidos para o domínio de interesse da ontologia a ser construída. Em outras palavras, deve-se considerar o reuso de ontologias já existentes.

O terceiro passo compreende a enumeração dos termos importantes para a ontologia, com o objetivo principal de conseguir uma lista abrangente dos termos relativos à ontologia, não se preocupando se os conceitos que eles representam estão sobrepostos ou se esses conceitos são classes, propriedades ou relações, como afirmam Noy e McGuinness (2000).

Após a obtenção da lista dos termos relevantes, passou-se para a definição das classes e da hierarquia de classes para a ontologia, construídos a partir dos termos obtidos no passo anterior. O método 101 permite que a construção da hierarquia de classe seja feita usando a abordagem 
Ontologia de aplicação no domínio de mortalidade: uma ferramenta de apoio para o preenchimento da declaração de óbitos

TABELA 1

Ontologias de aplicação na área de saúde

\begin{tabular}{|c|c|c|c|}
\hline Ontologia & Metodologia & $\begin{array}{l}\text { Ferramenta } \\
\text { de edição }\end{array}$ & Descrição \\
\hline $\begin{array}{l}\text { Ontologia de } \\
\text { referência para o } \\
\text { domínio da } \\
\text { cardiologia. }\end{array}$ & $\begin{array}{l}\text { EVOLUI } \\
\text { (ALVES, 2003) }\end{array}$ & $\begin{array}{l}\text { Não } \\
\text { especificada }\end{array}$ & $\begin{array}{l}\text { Define conceitos, subclasses e instâncias em cada uma das } \\
\text { subteorias do domínio cardiologia: patologia, anatomia do } \\
\text { coração, diagnóstico, terapia, avaliação clínica e exames } \\
\text { complementares. } \\
\text { (OLIVEIRA, 1999) }\end{array}$ \\
\hline $\begin{array}{l}\text { Ontologia para } \\
\text { sistemas de } \\
\text { emergência: } \\
\text { SAMU e Corpo } \\
\text { de Bombeiros. } \\
\text { Ontologia } \\
\text { referente às } \\
\text { doenças } \\
\text { sexualmente } \\
\text { transmissíveis } \\
\text { (DST) }\end{array}$ & Methontology & Protégé & $\begin{array}{l}\text { Ontologia para facilitar o reuso e provável integração futura } \\
\text { entre os sistemas de emergência em hospitais, clínicas e } \\
\text { aplicações de prontuário eletrônico. Pode ser usada no } \\
\text { desenvolvimento de sistemas baseados em conhecimento. } \\
\text { (MANICA, DANTAS e TODESCO, 2008) } \\
\text { Ontologia para gerenciar o conhecimento referente a Acquired } \\
\text { Immunodeficiency Syndrome (AIDS), Human Papiloma Virus } \\
\text { (HPV), sífilis, sífilis congênita, gonorréia, herpes genital, } \\
\text { linfogranuloma venéreo e tricomoníase. (FARIAS, MATTOS } \\
\text { e SIMÕES, 2003) }\end{array}$ \\
\hline $\begin{array}{l}\text { Ontologia } \\
\text { SODOnt }\end{array}$ & Método 101 & Protégé & $\begin{array}{l}\text { Define um vocabulário comum para médicos e um conjunto } \\
\text { de dados com suas estruturas para softwares que precisam } \\
\text { compartilhar informações de segunda opinião diagnóstica゙. } \\
\text { (PIRES, HALAH e RUIZ, 2006) }\end{array}$ \\
\hline $\begin{array}{l}\text { Ontologia para o } \\
\text { atendimento } \\
\text { emergencial de } \\
\text { pacientes }\end{array}$ & $\begin{array}{l}\text { OntoKen } \\
\text { (Ontologies for } \\
\text { Knowledge } \\
\text { Engineering and } \\
\text { Management) }\end{array}$ & Protégé & $\begin{array}{l}\text { Ontologia para troca de informações entre sistemas de } \\
\text { informação hospitalar em atendimento de urgência a } \\
\text { pacientes, englobando um vocabulário comum entre sistemas } \\
\text { heterogêneos para o compartilhamento de informações } \\
\text { relevantes sobre emergências. (LOPES, ANDRADE e VON } \\
\text { WANGENHEIM 2007) }\end{array}$ \\
\hline $\begin{array}{l}\text { Ontologia do } \\
\text { cartão nacional } \\
\text { de saúde }\end{array}$ & Não especificada & $\begin{array}{l}\text { Não } \\
\text { especificada }\end{array}$ & $\begin{array}{l}\text { Representa conceitos e relacionamentos definidos pelo } \\
\text { padrão de troca de mensagens do Sistema do Cartão } \\
\text { Nacional de Saúde. Engloba dados como os do atendimento } \\
\text { do paciente, tipos de alta médica, entre outros. } \\
\text { (NARDON e MOURA Jr., 2003) }\end{array}$ \\
\hline
\end{tabular}

* Segunda opinião diagnóstica ou médica constitui o parecer de outro médico, além do médico assistente, com o objetivo de auxiliar quanto à melhor conduta diagnóstica e às melhores opções de tratamento no caso clínico apresentado pelo paciente.

top-down ou a abordagem bottom-up ou ainda uma combinação das duas. Neste trabalho, foi adotada a abordagem top-down.

No quinto passo, foram definidas as propriedades das classes (slots) anteriormente criadas, baseadas nas informações extraídas do atual modelo do formulário da DO. Com base nessas informações, também foram definidas as restrições ou facetas (facets) dos slots - sexto passo - que incluem, por exemplo, restrições como tipos de dados, valores permitidos e cardinalidade, conforme previsto no método 101.

Ci. Inf., Brasília, DF, v. 39 n. 3, p.23-34, set./dez., 2010
O sétimo e último passo previsto no método corresponde à criação de instâncias para as classes definidas, preenchendo suas propriedades e atendendo às restrições definidas para cada propriedade. Neste passo, foram criadas instâncias a partir dos exemplos de registros de óbitos contidos no Manual de Instruçōes para o Preenchimento da Declaração de Óbitos.

Sobre as etapas realizadas para o desenvolvimento, a primeira corresponde à construção de uma ontologia preliminar no domínio de mortalidade, a partir dos conhecimentos adquiridos na consulta 
aos campos presentes no formulário da $\mathrm{DO}$ e seus relacionamentos, além dos documentos de referência do Ministério da Saúde com instruções de preenchimento da DO.

$\mathrm{Na}$ segunda etapa, com o suporte fornecido por profissionais de saúde da Secretaria Municipal de Saúde de Belo Horizonte (SMSA - BH), especializados no domínio pretendido, a ontologia desenvolvida na primeira etapa foi avaliada e readequada. A partir desse processo, foi desenvolvida uma nova ontologia sobre mortalidade, englobando conceitos e relacionamentos relevantes para o correto preenchimento da DO por parte dos médicos, fundamentada no conhecimento especializado do domínio.

$\mathrm{Na}$ primeira etapa, foram executados os sete passos definidos no Método 101 para a construção de uma ontologia e a inclusão de passos próprios da metodologia aqui adotada, tal como a utilização do método Sphere- $M^{6}$, conforme apresentado na figura 1.

A figura 1 mostra que o método Sphere- $M$ foi utilizado para auxiliar na captura dos termos da ontologia durante a execução do terceiro passo e também mostra o uso do editor Protégé na execução do quarto, quinto, sexto e sétimo passos, como forma de garantir maior agilidade, consistência e reusabilidade no processo de desenvolvimento.

Como resultado da execução dos passos realizados na primeira etapa, foi possível obter uma "hierarquia de conceitos" para a ontologia, que foi representada por meio da linguagem Ontology Web Language $(\mathrm{OWL})^{7}$ - obtida através de exportação no Protégé. Além disso, tornou-se possível realizar consultas à base de conhecimento da ontologia utilizando as queries construídas com o auxílio do Protégé.

Utilizando os conhecimentos apreendidos na etapa anterior, porém não reutilizando os termos definidos anteriormente, optou-se pelo desenvolvimento de

\footnotetext{
${ }^{6}$ Sphere-M é um método que busca orientar a tarefa de captura de uma ontologia, através de passos, regras e métricas baseadas em um algoritmo implementado para realizar a busca em profundidade em um grafo. (ALENCAR e ZÁRATE, 2007).

${ }^{7}$ Linguagem padrão para expressar ontologias do W3 Consortium. Sintaxe disponível no endereço: http://www.w3c.org
}

uma nova ontologia na segunda etapa do trabalho, que contou com a participação e a colaboração dos profissionais de saúde e epidemiologia da SMSA$\mathrm{BH}$, diferentemente do que ocorreu na primeira etapa. A participação desses profissionais no desenvolvimento e validação da ontologia permitiu fundamentar os conceitos e relacionamentos definidos em razão da experiência prática cotidiana dessas pessoas no domínio tratado.

$\mathrm{Na}$ segunda etapa, o desenvolvimento da ontologia definida como "ontologia sobre o preenchimento da Declaração de Óbitos" foi realizado utilizandose o Método 101, com pequenas alterações, como é mostrado pela figura 2.

Foram executados seis dos sete passos previstos no Método 101, conforme mostra a figura 2. O sétimo passo ("criar instâncias") deste método foi substituído pelo passo "validar ontologia", que também está contido nele, porém não é apresentado de forma explícita. A etapa de validação é aqui apresentada como um passo explícito, devido à sua importância para o desenvolvimento e aplicação da ontologia sobre o preenchimento da DO.

A atividade de "criar instâncias" não foi necessária à ontologia desenvolvida, porque a definição das classes hierarquicamente, das propriedades e das restrições tornou-se suficiente para atender o nível de especificação de conhecimento requerido para o preenchimento da $\mathrm{DO}$, o que não exclui a possibilidade de realizar essa instanciação no constante processo de evolução da ontologia.

A figura 2 mostra também que a etapa de validação representa um processo iterativo no desenvolvimento da ontologia, exercendo fortes influências na execução do terceiro ao sexto passos, quando alguns dos termos definidos antes da validação tiveram de ser redefinidos.

A participação de epidemiologistas da SMSA-BH na enumeração dos termos para a ontologia - terceiro passo - e a sua colaboração para o processo de validação - sétimo passo - também estão representados na 
Arquitetura de desenvolvimento da $1^{a}$ etapa: ontologia preliminar

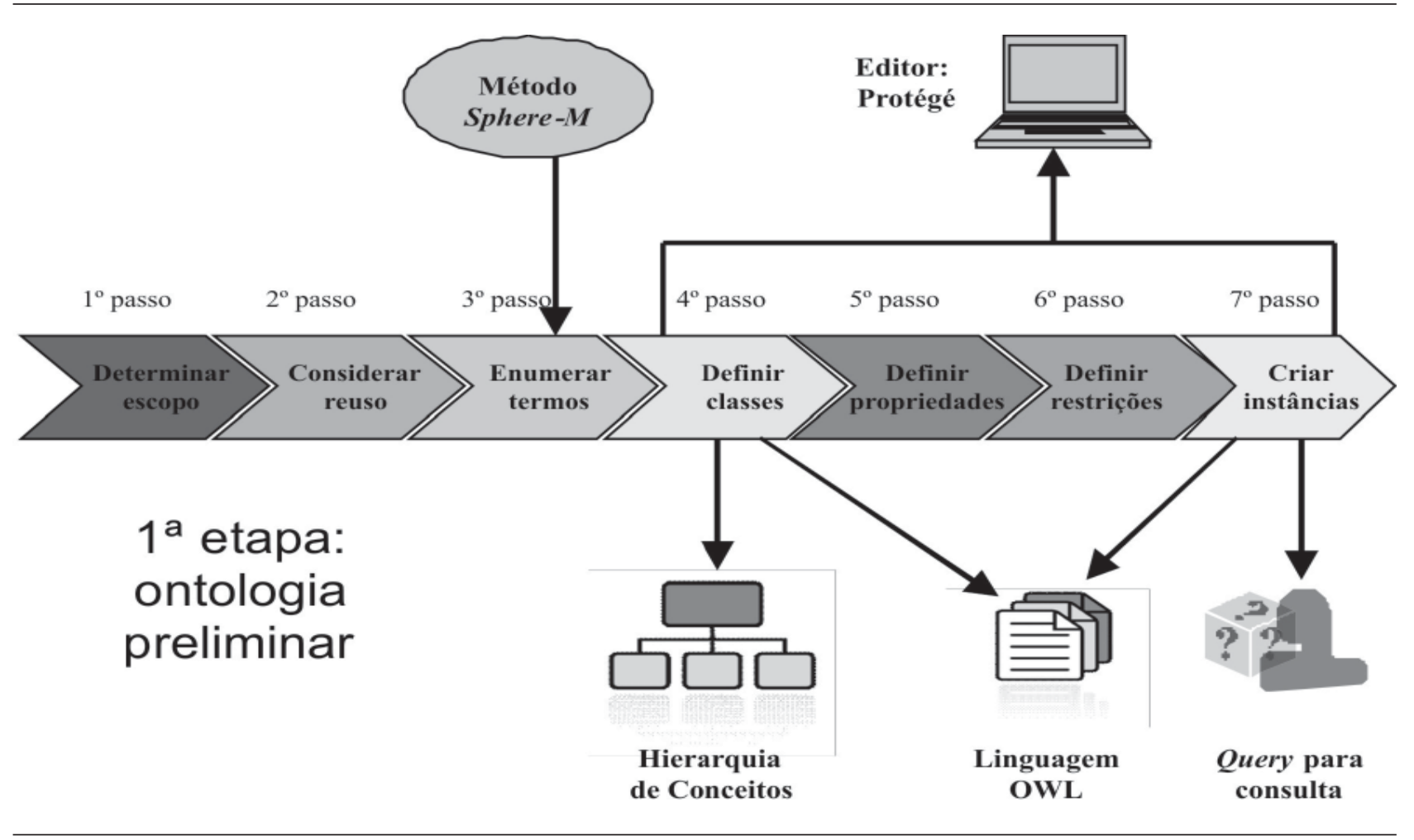

FIGURA 2

Arquitetura de desenvolvimento da $2^{\circ}$ etapa: ontologia sobre o preenchimento da DO

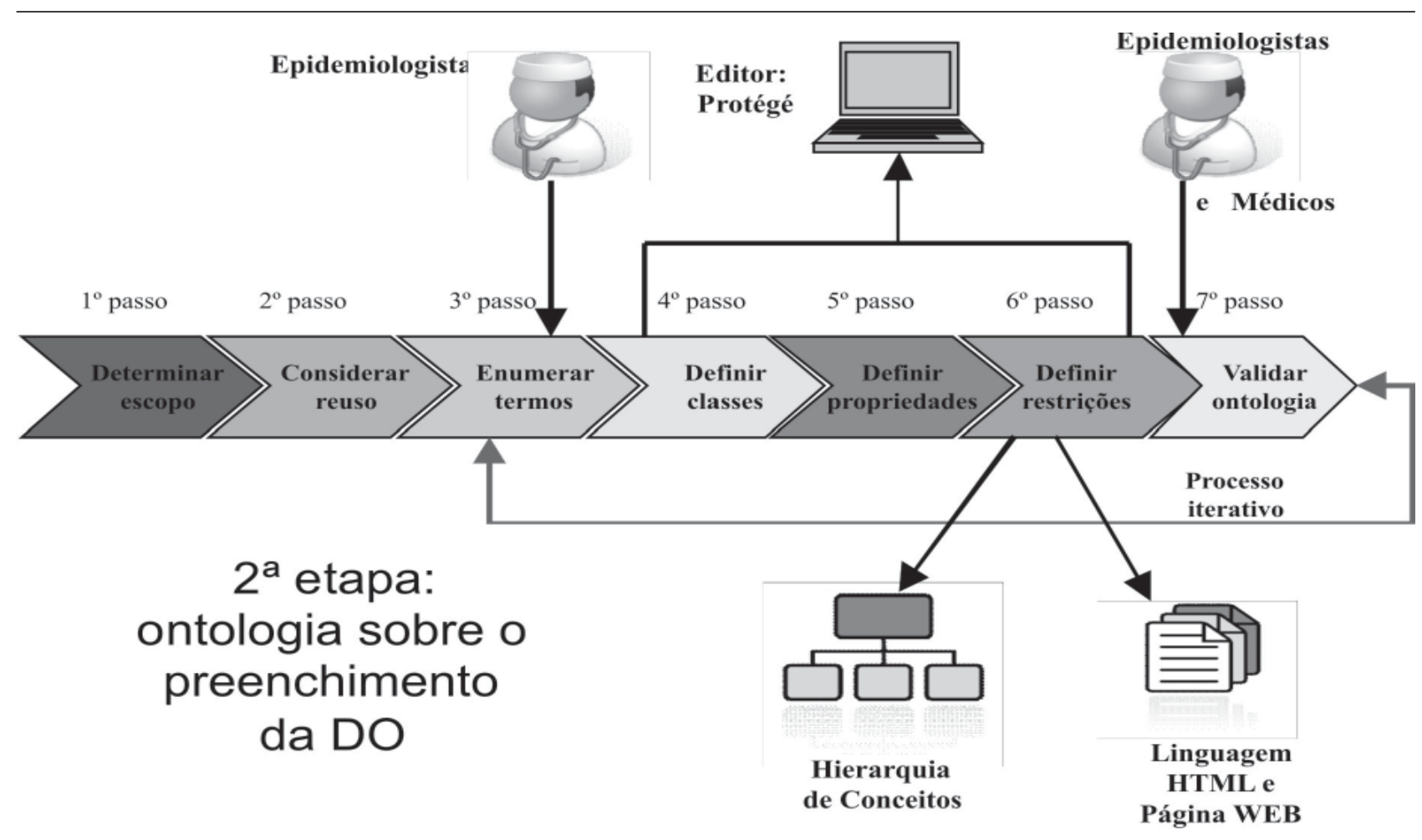


figura 2. Foram realizados grupos focais ${ }^{8}$ com estes profissionais para a validação da ontologia, tendo por objetivo coletar as diferentes percepções sobre os conceitos do domínio de mortalidade.

\section{RESULTADOS}

Os passos realizados na metodologia adotada permitiram gerar uma ontologia no domínio de mortalidade contendo os termos que os médicos necessitam conhecer para declarar óbitos no formulário e o relacionamento semântico entre esses termos.

Os termos definidos representam os conceitos gerais que os médicos necessitam conhecer para declarar óbitos no formulário, tais como as circunstâncias do óbito (morte natural ou morte não natural), a categoria do falecido (feto ou não feto, menor de um ano, homem, ou mulher) e os campos de preenchimento obrigatório para cada tipo de óbito ocorrido, conforme mostra a figura 3.

Como resultado da execução dos passos realizados na segunda etapa da metodologia, foi possível obter uma nova "hierarquia dos conceitos" para ontologia, representada por meio da linguagem HTML, gerada através de exportação no Protégé (figura 2). Embora a linguagem HTML não ofereça o recurso de representar o conhecimento contido na ontologia, ela foi útil como o passo inicial para a construção de um site, utilizado para apresentar e disponibilizar a base de conhecimento da ontologia sobre o preenchimento da DO aos médicos.

Partindo do código HTML gerado pelo Protégé, foi construído um site para acesso à base de conhecimento da ontologia, em que a página inicial (index.html) apresenta a Hierarquia de Conceitos, representada através de hiperlinks dispostos em uma estrutura taxonômica (figura 4, a seguir). $\mathrm{O}$ acesso ao site pode ser feito pelo do endereço: http:// www.ontologiamortalidade.gratix.com.br/, que está disponibilizado na web para consulta pelos médicos, inicialmente em um domínio temporário.

\footnotetext{
${ }^{8}$ Segundo Morgan (1997), grupos focais podem ser definidos "como uma técnica de pesquisa para coleta de dados por meio das interações grupais ao se discutir um tópico especial sugerido pelo pesquisador".
}

\section{FIGURA 3}

\section{Hierarquia de classes da ontologia sobre o preenchimento da DO}

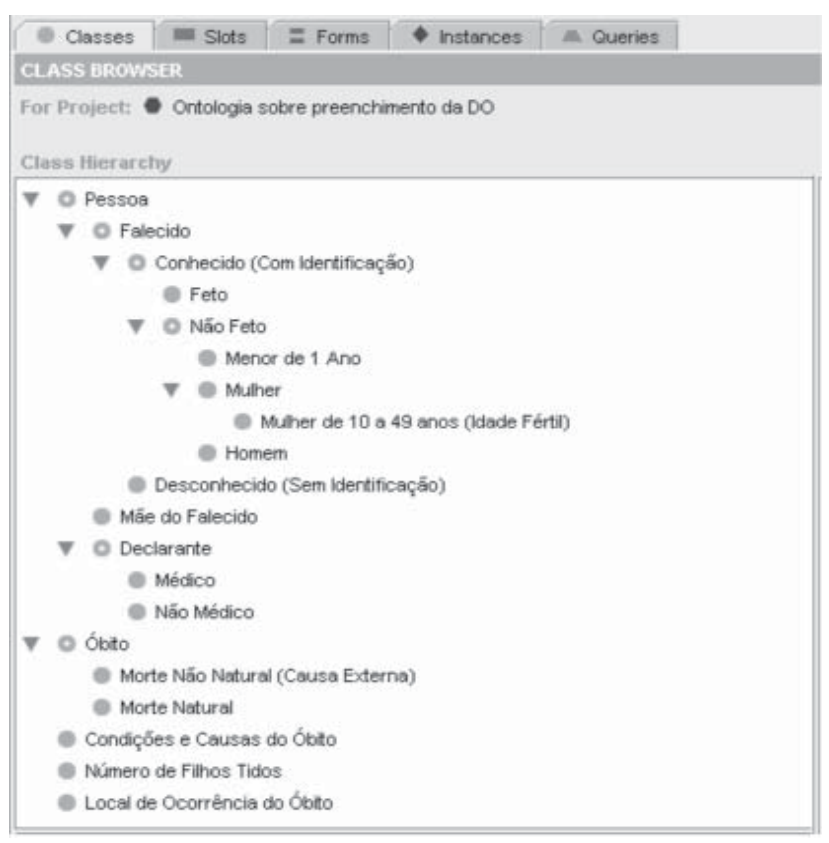

Para cada um dos conceitos que sejam classes concretas nesta hierarquia, criou-se um biperlink para acesso à página criada para ele. A página de cada conceito contém sua descrição, suas propriedades (slots), suas restrições (facets), hiperlinks para acesso às propriedades que representam relacionamentos e hiperlinks para as classes-pai e classes-filho correspondentes (estrutura taxonômica).

Na página referente a um conceito específico, o médico pode consultar as informações sobre tal conceito e utilizá-las ao preencher a DO. Uma das possíveis consultas pode ser feita, por exemplo, à página do conceito Feto, para que o médico possa visualizar quais os campos devem ser preenchidos para este tipo de óbito, e como deve ser esse preenchimento (figura 5, a seguir).

A página sobre o conceito Feto contém uma descrição sobre esse conceito, a classe-pai (ou Nível Superior) Conhecido, nenhuma classe-filho (ou Nível Inferior), as propriedades (slots) da classe Feto: 11. Nome do Falecido, 13. Nome da Mãe, 14. Data de Nascimento, 15. Idade, 16. Sexo, 41. Peso, Dados do Óbito e Dados da Mãe, além das restrições 
Ontologia de aplicação no domínio de mortalidade: uma ferramenta de apoio para o preenchimento da declaração de óbitos Figura 4

Página principal para acesso à ontologia sobre o preenchimento da DO

\section{Ontologia sobre Mortalidade}

\section{Hierarquia de Conceitos para o Preenchimento da Declaração de óbitos}

\section{Autores: Fabrício Martins Mendonça, Eliane Drumond e Ana Maria Cardoso}

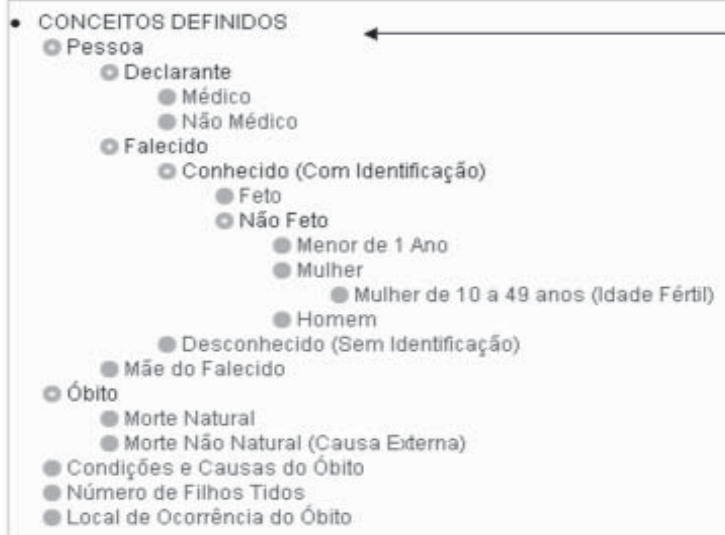

FIGURA 5

Página que apresenta o conceito Feto da ontologia de preenchimento da DO

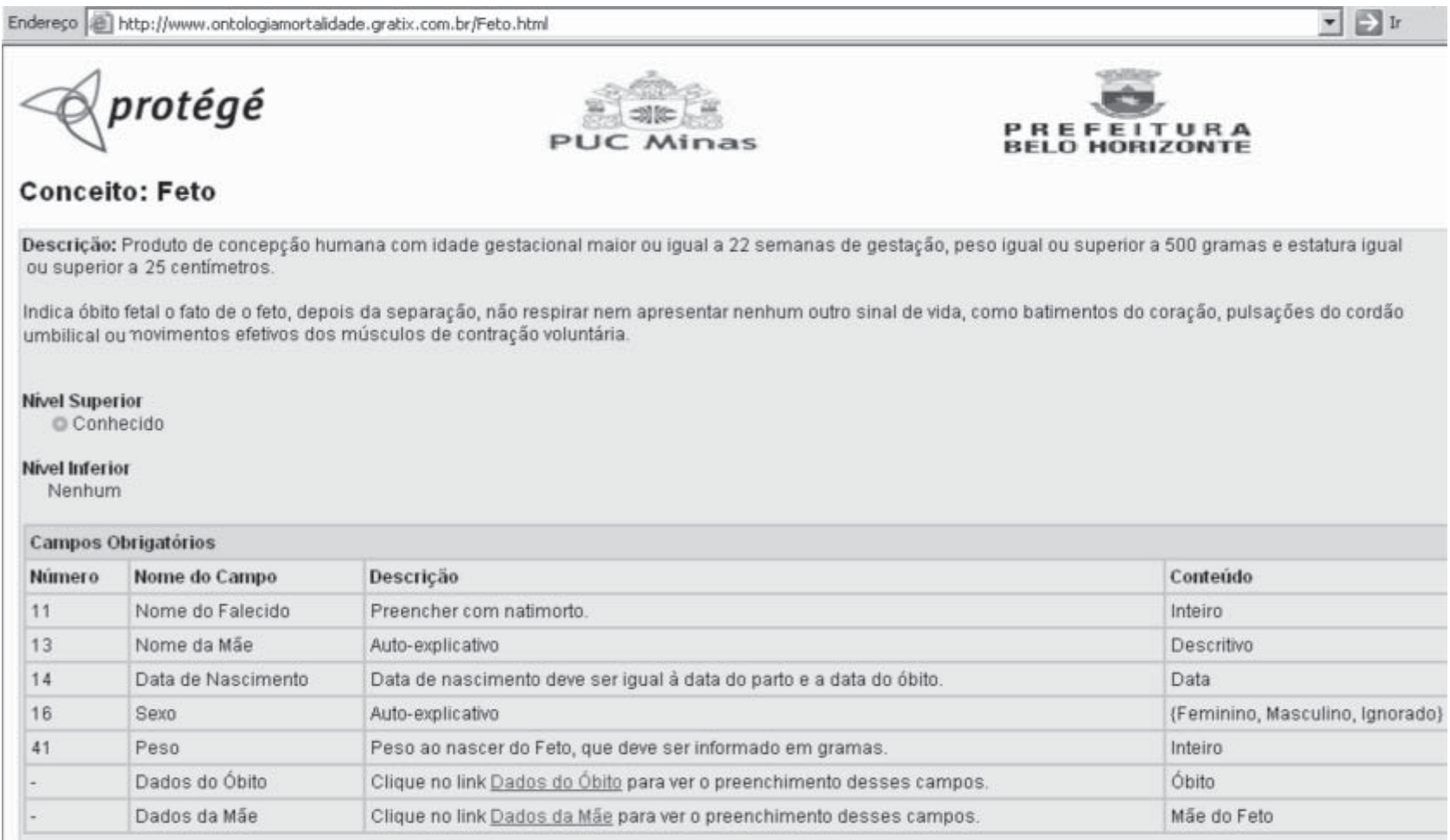


(facets) dessas propriedades, apresentadas na coluna Conteúdo: Inteiro, Descritivo, Data, \{Feminino, Masculino, Ignorado\}, entre outras.

As propriedades Dados do Óbito e Dados da Mãe, que representam relacionamentos entre classes, possuem hiperlinks para acesso à página de cada uma dessas classes, respectivamente Óbito.btml e Mãe_do_Falecido.btml. Esse recurso permite visualizar os demais campos de preenchimento obrigatório na DO, quando o óbito é fetal.

\section{CONCLUSÕES E TRABALHOS FUTUROS}

O uso de ontologias como um artefato computacional mostrou-se adequado para representação do conhecimento sobre um domínio específico em saúde-mortalidade-com o propósito de representar o conhecimento necessário ao correto preenchimento da DO por parte dos médicos.

O processo de desenvolvimento de ontologias apresentou-se como um percurso metodológico adequado aos propósitos deste trabalho, embora encontrando obstáculos inerentes à construção de ontologias para descrever o conhecimento de domínios específicos.

Uma das primeiras dificuldades é a inexistência de um modelo padrão único. Neste trabalho, optou-se pelo método 101 e o editor de ontologias Protégé, tendo em vista a relação de proximidade existente entre o software e o método e a utilização recorrente de ambos na construção de ontologias.

Outro problema decorre da necessidade de grande conhecimento sobre o domínio tratado, contornada pelo apoio de profissionais da SMSA-BH especialistas no tema. Esse apoio permitiu o amadurecimento da ontologia; no entanto, cabe considerar que dentro de um domínio amplo, tal como o de mortalidade, dificilmente será atingido um conhecimento consensual. Isso justifica o fato de a construção de uma ontologia ser um processo iterativo, e ao longo do tempo apresentar evoluções na sua base de conhecimento.

Paralelamente à evolução da ontologia, melhorias ao atual site de acesso à sua base de conhecimento podem ser incorporadas com o objetivo de propiciar maior interatividade do médico com os conceitos presentes nesta base. Entre essas melhorias podese citar a criação de uma interface de busca aos termos representativos de conceitos na ontologia e a inclusão de uma função que permita ao próprio médico sugerir novos conceitos, ajudando ainda mais a incrementar o conhecimento que ela representa.

Com relação à evolução da ontologia, mostra-se pertinente a inclusão de termos para representar o conhecimento das possíveis causas de morte que podem provocar o óbito, previstas no diretório da Décima Classificação Internacional de Doenças (CID-10). A ontologia, então, deverá ser capaz de representar o conhecimento relativo às informações que são declaradas no campo 49 (Bloco VI) da $\mathrm{DO}$, que corresponde às causas de morte que provocaram o óbito, estejam elas na Parte I (causas consequenciais ou intermediárias) ou na Parte II (causas contribuintes) (BRASIL, 2001).

Para realizar essa proposta, torna-se necessário delimitar o escopo que a ontologia irá cobrir, definindo os grupos de causas de morte que serão representados. Essa é uma estratégia racional, ante a abrangência de causas de morte existentes no vocabulário médico ou no repertório da CID-10.

Diante do contexto abrangente em que se insere uma ontologia sobre o domínio de mortalidade, pode-se afirmar que a ontologia aqui relatada está em fase de evolução e necessita de aprimoramentos. Esta constatação é usual no processo de criação de uma ontologia, que se caracteriza como atividade iterativa realizada por uma sequência de passos, e que deve sempre ser reavaliada.

Essa é uma medida acertada para que cada vez mais a ferramenta computacional apresentada possa trazer contribuições a médicos e outros profissionais de saúde envolvidos com o registro de óbitos no Brasil. O artigo representa uma pequena contribuição, dentre tantas outras que devem ser feitas, para trazer melhorias à qualidade da informação sobre mortalidade produzida em nosso país.

Artigo submetido em 08/11/2010 e aceito em 24/05/2011. 
Ontologia de aplicação no domínio de mortalidade: uma ferramenta de apoio para o preenchimento da declaração de óbitos

\section{ANEXO I - ATUAL MODELO DO FORMULÁRIO DA DO}

FIGURA 6

Atual modelo do formulário da DO

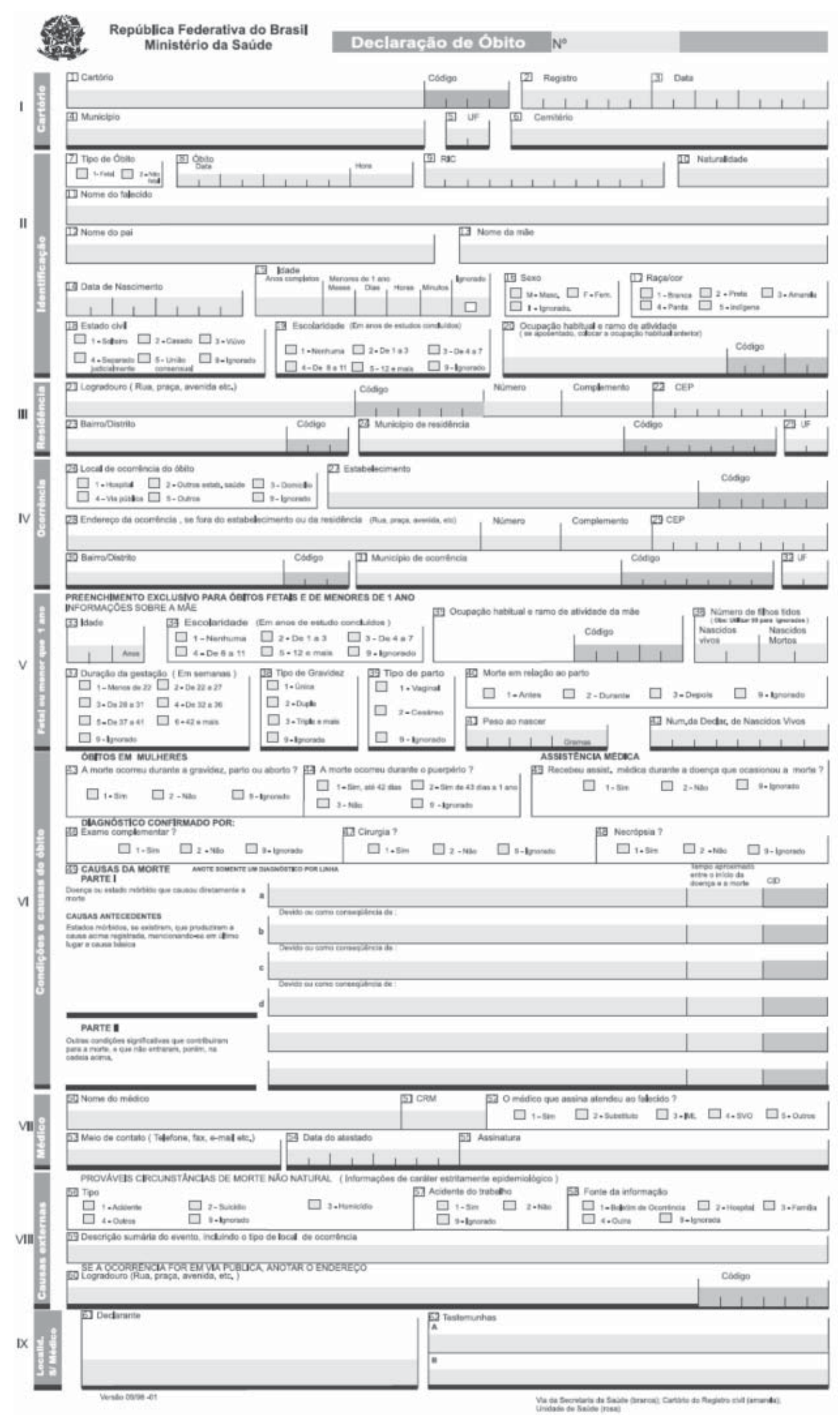




\section{REFERÊNCIAS}

ALENCAR, R. O.; ZÁRATE, L. E. Proposal of a method to assist the ontological study of domains. XVIII In: SIMPÓSIO BRASILEIRO DE INFORMÁTICA NA EDUCAÇÃO, 18., 2007. Anais. Disponível em: < http://www.br-ie.org/pub/index.php/sbie/ article/view/558>. Acesso em: 6 jul. 2011.

ALMEIDA, M. B.; BAX, M. P. Uma visão geral sobre ontologias: pesquisa sobre definições, tipos, aplicações, métodos de avaliação e de construção. Ciência da Informação, v. 32, n. 3, p. 7-20, 2003.

ALVES, J. W. V. EVOLUI : uma metodologia para auxiliar a criação de ontologias. 2003. Dissertação. (Mestrado em Informática Aplicada) Universidade de Fortaleza, Fortaleza, 2003.

BRASIL. Ministério da Saúde. Fundação Nacional de Saúde. Manual de instruções para o preenchimento da declaração de óbito. Brasília, 2001. Disponível em: < http://www.unirio.br/hugg/arquivos/declaracao_ obitos\%20.pdf>. Acesso em: 6 jul. 2011.

BRASIL. Ministério da Saúde; Conselho Federal de Medicina. A Declaração de Óbito: documento necessário e importante. Brasília: Ministério da Saúde, 2006. Disponível em: < http://www.portalmedico.org.br/ arquivos/cartilha_do_cfm_ms.pdf>. Acesso em: 6 jul.2011.

ELUAN, A. A.; FACHIN, G. R. B. Ontologia para publicação cientifica. Disponível em: < http://www.uff.br/ontologia/artigos/316.pdf.>. Acesso em: 7 jul. 2011.

FARIAS, R. F.; MATTOS, M. C.; SIMÕES, P. W. T. A. Ontologia para a gestão do conhecimento em saúde por meio da metodologia Methontology. Disponível em: http://www.sbis.org.br/cbis/arquivos/857.pdf. Avesso em: 7 jul. 2011.

GÓMEZ-PÉREZ, A. Evaluation of taxonomic knowledge in ontologies and knowledge bases. In: PROCEEDINGS OF THE BANFF KNOWLEDGE ACQUISITION FOR KNOWLEDGEBASED SYSTEMS WORKSHOP. KAW'99. Disponível em: < http://oa.upm.es/6456/1/Evaluation_of_Taxonomic_K.pdf $>$. Acesso em: 7 jul. 2011.

GONDIM, S. M. G. Grupos Focais como técnica de investigação qualitativa: desafios metodológicos., Salvador: UFba, 2002.

GRUBER, T. R. Towards principles for the design of ontologies used for knowledge sharing. Disponível em: < http://itee.uq.edu.au/ infs3101/_ Readings/OntoEng.pdf> Acesso em: 7 jul. 2011.
GUARINO, N. Formal Ontology in Information Systems. In: FOIS'98,1998, Trento, Italy. Proceedings....Amsterdam: IOS Press,1998. p. 3-15. Disponível em: < http://citeseerx.ist.psu.edu/ viewdoc/download?doi=10.1.1.29.1776\&rep=rep1\&type $=$ pdf $>$. Acesso em: 8 jul. 2011.

LOPES, P. M. A.; ANDRADE, R. e VON WANGENHEIM, A. Uma ontologia para o atendimento emergencial de pacientes. Disponível em: < http://www.sbis.org.br/cbis11/arquivos/832.pdf>. Acesso em: 7 jul. 2011.

MANICA, H.; DANTAS, M. A. R. e TODESCO, J. L. Ontologia para compartilhamento e representação de conhecimento em saúde. Diálogos \& Saberes, Mandaguari, v. 4, n. 1, p. 151-161, 2008.

MENDONÇA, F. M. Ontologia de aplicação no dominio de mortalidade: uma ferramenta de apoio para o preenchimento da Declaração de Óbitos. 2009. Dissertação (Mestrado em Informática). Pontifícia Universidade Católica de Minas Gerais, Programa de Pós-graduação em Informática. Belo Horizonte, 2009.

MORGAN, D. Focus group as qualitative research. London: Sage Publications, 1997. (Qualitative Research Methods Series, 16)

NARDON, F. B. e MOURA Jr., L. A. Ontologias e banco de dados dedutivos para integração de informações em saúde. Disponível em: < http://telemedicina.unifesp.br/pub/SBIS/CBIS2004/trabalhos/ arquivos/348.pdf> Acesso em: 8 jul. 2011.

NIOBEY, F. M. L.; CASCÃO, A. M.; DUChIADE, M. P.. BARBOZA, P. C. Qualidade do preenchimento de atestados de óbitos de menores de um ano na região metropolitana do Rio de Janeiro. Rev. Saúde pública, São Paulo, v. 24, n. 4, p. 311-8, 1990. Disponível em: < http://www.scielosp.org/pdf/rsp/v24n4/09.pdf>. Acesso em: 7 jul. 2011.

NOY, N. F.; MCGUINNESS, D. L. Ontology Development 101: A guide to creating your first ontology. Disponível em: $<$ http:/ / citeseerx.ist. psu.edu/viewdoc/download?doi=10.1.1.136.5085\&rep=rep1\&type $=$ pdf $>$. Acesso em: 7 jul. 2011.

OLIVEIRA, K. M. Modelo para Construşão de Ambientes de Desenvolvimento de Software Orientados a Dominio. 1999. Tese (Doutorado em Engenharia e Sistemas de Computação) UFRJ. Rio de Janeiro.

PIRES, D. F.; HALAH, R. A. e RUIZ, E. E. S. Interoperabilidade semântica na troca de informações de segunda opinião diagnóstica. Disponível em: < http://www.sbis.org.br/cbis/arquivos/885.pdf>. Acesso em: 7 jul. 2011. 